\title{
Aktivierende Sozialpolitik zwischen Systemimperativ und Eigensinn: Eine Untersuchung der Effekte und Aneignungen der Bedarfsorientierten Mindestsicherung in Österreich
}

\author{
Claudia Globisch · Fabian Madlung
}

(C) Der/die Autor(en) 2017. Dieser Artikel ist eine Open-Access-Publikation.

Zusammenfassung Der Beitrag untersucht die Effekte und Aneignungsformen aktivierender Sozialpolitik am Beispiel der Bedarfsorientierten Mindestsicherung in Österreich. Grundlage hierfür ist eine umfassende qualitative Studie auf Basis von narrativen Interviews mit Mindestsicherungsempfänger_innen und Berater_innen, die zwischen 2013 und 2015 erhoben wurden. ,Aktivierung“ wird dabei als normative Vorgabe und zugleich institutionalisierte Strategie rekonstruiert, die mit Hilfe unterschiedlicher Interventionen und Programme auf die Formierung von Einstellungen und Verhaltensmustern zur Herstellung marktgängiger Subjekte und ihrer „Employability“ zielt und als Herrschafts- und Selbsttechnologie verstanden wird. Die Frage, ob sich Subjekte gemäß den sozialpolitischen Zielen der ,employablity“ aktivieren lassen, wird mit einer Typologie von Handlungsaktivierungen beantwortet, die zeigt, dass die untersuchten Subjekte zwar in den meisten Fällen ,,aktiv“, aber nicht durch die aktivierungspolitischen Maßnahmen im Sinne der „employability“ aktiviert werden. Die Überlegungen stehen im Spannungsfeld zwischen Armuts- und Arbeitslosigkeitsforschung, Subjektivierung und Gouvernementalitäts-Forschung.

Schlüsselwörter Aktivierung · Sozialpolitik · Gouvernementalität ·

Subjektivierung · Arbeitslosigkeit

\footnotetext{
C. Globisch $(\bowtie) \cdot$ F. Madlung

Institut für Soziologie, Universität Innsbruck, Universitätsstraße 15, 6020 Innsbruck, Österreich

E-Mail: Claudia.Globisch@uibk.ac.at

F. Madlung

E-Mail: Fabian.Madlung@student.uibk.ac.at
} 


\title{
Activating social policy between system imperative and self- determination: A study on the effects and adoption of Austrian's needs- based minimum benefit system
}

\begin{abstract}
Taking Austrian's needs-based minimum benefit system as an example, the article examines the effects and the adoption of activating social policy. The contribution is based on a qualitative study using narrative interviews with both recipients of the needs-based minimum benefit and case managers, held between 2013-2015. We reconstruct "activation" as normative requirement and institutionalized strategy that aims to shape attitudes and behaviour in order to construct marketable and employable subjects. In order to answer the question, whether and how subjects can be activated according to the socio-political aim of "employability", we propose a typology of "action activation", showing that, in general, most people are indeed "active", though not activated through and according to the aims of "employablity". Our considerations are located on the intersections of research on poverty and unemployment, research on subjectivation and governementality studies.
\end{abstract}

Keywords Activation - Social policy - Governmentality - Subjectivation · Unemployment

\section{Mit Aktivierungspolitik aus der Armutsfalle und der Krise des Wohlfahrtsstaates?}

Durch Sozialpolitik vermittelter sozialer Ausgleich zwischen den ungleich verteilten Macht- und Marktchancen von Arbeit und Kapital - das war die Idee beim Aufbau wohlfahrtsstaatlicher Regime in den westlich geprägten, marktwirtschaftlich organisierten Industrienationen nach dem Zweiten Weltkrieg (vgl. Lessenich 2008, S. 59 ff.; Streeck 2013). Die individuellen Folgen und Risiken von marktwirtschaftlichen Krisen und konjunkturzyklischer Arbeitslosigkeit, die insbesondere die lohnabhängig Beschäftigten sowie den nicht (mehr) arbeitenden Teil der Bevölkerung betrafen, wurden durch eine zumindest bedingte Entkoppelung von Wohlfahrtsproduktion und Lebensschicksal der Einzelnen vom Arbeitsmarkt unter anderem durch staatliche Konjunkturprogramme und wohlfahrtsstaatliche Institutionen der Sozialversicherungen abgefedert. Grundsätzlich blieben die sozialstaatlichen Sicherheitsleistungen aber immer an nachgewiesene Arbeitsbereitschaft geknüpft (vgl. Vobruba 2006), eine bedingungslose Entkopplung von Arbeit und Einkommen erfolgte nicht. Dennoch wurde das Verhältnis zwischen Wohlfahrtsstaat und Subjekt in den Sozialwissenschaften bis Mitte des 20. Jahrhunderts als primär befähigendes und autonomieermöglichendes beschrieben (vgl. Vobruba 1989; Dux 2008, 2009; Lessenich 2008).

Dieses wohlfahrtsstaatliche Modell ist seit den 1970er-Jahren zunehmend in die Krise geraten (vgl. Habermas 1998; Streeck 2013), muss es mit der Verknüpfung von Arbeit und Einkommen doch immer schon zwei Steuerungsprobleme bewältigen: ,de[n] Transfer von Geld aus dem ökonomischen System in das System sozialer Sicherung“ (Vobruba 2006, S. 32) einerseits sowie die Unterbindung der „Ausbrei- 
tung von Motiven zu dauerhafter arbeitsmarktexterner Lebensführung“ (ebd., S. 33) andererseits. In ökonomischen Krisen, die sich mit dem Übergang von fordistischen zu postfordistischen Produktionsverhältnissen in zunehmenden Privatisierungs-, Flexibilisierungs- und letztlich auch Finanzialisierungstendenzen ausdrücken, wird der Geldtransfer problematisch und die ökonomischen Krisen werden meist auch zu Krisen des Sozialstaats (ebd.).

Auf die vielfältigen Krisenerscheinungen des modernen Kapitalismus, die unter anderem in einem Anstieg der (Langzeit-)Arbeitslosigkeit in Erscheinung getreten sind, haben die westlich geprägten Wohlfahrtsstaaten in verschiedener Weise und in unterschiedlichem Tempo reagiert. Auch wenn nicht von einer harmonisierten und europäisierten Sozialpolitik die Rede sein kann, gibt es dennoch europäische Rahmenbedingungen und Vorgaben, die die Ausgestaltung nationaler Sozialpolitik figurieren. Zentral hierfür ist die im Jahr 2000 verabschiedete Lissabon-Strategie, die zum Ziel hatte, die Europäische Union innerhalb von zehn Jahren ,zum wettbewerbsfähigsten und dynamischsten wissensbasierten Wirtschaftsraum in der Welt zu machen“ (Europäischer Rat 2000). Sozialpolitik wurde dabei als Teil der europäischen Sozialagenda doppelt zweckbestimmt: „Die Agenda muss die Rolle der Sozialpolitik als Wettbewerbsfaktor stärken und ihr gleichzeitig eine effizientere Verfolgung eigener Ziele in Bezug auf den Schutz des Einzelnen, den Abbau von Ungleichheiten und den sozialen Zusammenhalt ermöglichen." (Rat der Europäischen Union 2001, S. 6) Konsequenz daraus war, die stärkere Beteiligung am Arbeitsmarkt zu forcieren, da aus Perspektive der Europäischen Kommission und des Rates der Europäischen Union ,,mehr und bessere Arbeitsplätze [. . .] der Schlüssel zur sozialen Eingliederung [sind].“ (ebd.) Die „Entwicklung eines aktiven und dynamischen Wohlfahrtsstaates“ (ebd.) wurde dabei als zentral herausgehoben, und dem Konzept der „Aktivierung“ wurde durch die Europäische Beschäftigungsstrategie (EBS) Nachdruck verliehen. Sie wurde von den Mitgliedsstaaten unterschiedlich interpretiert und umgesetzt (vgl. Klammer und Leiber 2004; Zirra 2010).

Dem Konzept der „Aktivierung“ liegt eine Kritik am alten (versorgenden) Sozialstaatsmodell zugrunde, die die sozialstaatlichen Systeme vornehmlich unter den Gesichtspunkten budgetierter Kosten und negativer Leistungsanreize thematisiert und von den passivierenden Effekten eines versorgenden Staates ausgeht, die arbeitslose TransferleistungsempfängerInnen zunehmend von gesellschaftlicher Teilhabe (gemeint war primär Arbeitsmarktintegration) ausschließen und marginalisieren würden. Mit dem Konzept verbunden ist ein Erklärungsmuster, das davon ausgeht, dass in allen Subjekten im Kern ein gleichermaßen von außen zu aktivierendes Potential angelegt sei und der Erfolg der Aktivierung maßgeblich von der Eigenaktivität abhängen würde (vgl. Wenzel 2008; Globisch 2012). Damit sollte die (Arbeitsmarkt-)Integration der Subjekte einerseits, die Kostenminimierung der Sozialsysteme andererseits zu erreichen sein. „Aktivierungspolitik“ soll demnach die Krise sozialstaatlicher Systeme lösen, indem die angenommene Passivität der Subjekte bearbeitet wird. Darauf gründen die in den einzelnen Ländern umgesetzten aktivierungspolitischen Instrumente und Maßnahmen, die mit dem Ziel angetreten sind, Subjekten aus der behaupteten Passivitätsfalle herauszuhelfen.

Arbeitslosigkeit wird mithin zunehmend nicht als Krise des Arbeitsmarktes, sondern vielmehr als Krise des Subjekts gedeutet und behandelt; Armut bewältigen 
heißt nunmehr, eine selbstverschuldete Lage selbsttätig zu lösen und dabei von einem Apparat, der „Fördern und Fordern“ zur Maxime macht, aktivierend und drängend, aber auch strafend und disziplinierend begleitet zu werden (vgl. auch Hirseland und Ramos-Lobato 2012). Nicht mehr die Gewährung von Schutzrechten, sondern die Verknüpfung individueller Anrechte mit Verpflichtungen zur eigenverantwortlichen Integration in die Arbeitsgesellschaft sind die Maxime der reformierten Sozialpolitik (vgl. Lessenich 2012; Bothfeld und Betzelt 2014).

Begleitet wird dieser Paradigmenwechsel von einer flächendeckenden Umgestaltung des Sozialstaates und seiner Institutionen zu neoliberalen Dienstleistungsagenturen, deren Auftrag sich nicht mehr an BürgerInnen, sondern an „KundInnen“ richtet. Die Sozialleistungen basieren dabei auf „,bedarfsgeprüften“, aber knapp bemessenen Leistungen, die einerseits an unbedingte Arbeitswilligkeit und dafür notwendige „Bewährungsbemühungen“ geknüpft sind und andererseits konditionalisiert sind, womit damit faktisch (qua Sanktionen, die auf Mittelkürzungen basieren) das Grundrecht auf ein Existenzminimum zur Disposition gestellt ist. Typisch für den gouvernementalen Doppelimperativ des „Forderns und Förderns“ steht die ambivalente Gleichzeitigkeit eines institutionellen Gegenübers, das sich als anleitender Partner und Berater vorstellt und Empowerment der „KundInnen“ verspricht, dabei aber andererseits mit Mitteln wie Anweisung und Sanktionierung ein asymmetrisches Machtverhältnis produziert. Dieser Umbau hin zu neoliberalen Regierungsformen mit dem Ziel der Herstellung eigenverantwortlicher, marktgängiger Subjekte steht im Kontext einer vieldiskutierten, umfassenden Transformation des Regierens und Regulierens, die als „Gouvernementalität“ diskutiert wird (vgl. Foucault 2010; Rose 1996; Lemke et al. 2000; Bröckling 2007; Scott und Weiskopf 2008; Le Galès und Scott 2009; du Gay und Scott 2010; Hibou 2015).

Mit Gouvernementalität meint Foucault den Zusammenhang von Herrschaftsund Selbsttechnologien, ,den Kontaktpunkt, an dem die Form der Lenkung der Individuen durch andere mit der Weise ihrer Selbstführung verknüpft ist" (Foucault 1993, S. 203, zitiert nach der Übersetzung von T. Lemke: Lemke et al. 2000, S. 29). Das Konzept der „Aktivierung“ lässt sich als eine ebensolche Technik beschreiben, mit der das Subjekt als durch Maßnahmen aktivierbar und individuell verantwortlich konstruiert und ihm eine kompetente Sorge um und Aktivierung von sich zuschreibt. „Im Rahmen neoliberaler Gouvernementalität signalisieren Selbstbestimmung, Verantwortung und Wahlfreiheit nicht die Grenze des Regierungshandelns, sondern sind selbst ein Instrument und Vehikel, das Verhältnis der Subjekte zu sich selbst und zu den anderen zu verändern“ (ebd., S. 30).

„Aktivierung“ ist eine normative Vorgabe und zugleich institutionalisierte Strategie, die mithilfe unterschiedlicher Interventionen und Programme auf eine Formierung von Einstellungen und Verhaltensmustern zur Herstellung marktgängiger Subjekte und ihrer „Employability“ zielt. Der neoliberale Wohlfahrtsstaat nimmt dabei die Rolle einer moralischen Instanz ein, um Subjekte zu Verhaltensänderung und Eigenverantwortung im Dienste der Gesellschaft zu ,,aktivieren“ und zu sich selbst ins Verhältnis zu setzen, d. h. ,,sinnhafte Handlungsformen der Selbstregierung“ zu initialisieren, „die insbesondere durch Techniken des Selbst (Foucault 1984) realisiert werden“ (vgl. Pfahl und Traue 2012). Ob dies gemäß den sozialpolitischen Zielen gelungen ist, d. h. sich Subjekte, die sich trotz ihrer Angewiesenheit auf Sozialleis- 
tungen in unterschiedlichen Lebenssituationen mit unterschiedlichen biografischen Hintergründen und Lebensplänen befinden, (mit dem Ziel der Arbeitsmarktintegration) ,aktivieren“ lassen (können), soll in diesem Beitrag diskutiert werden.

Dies geschieht am Beispiel der noch relativ unerforschten Realisierung aktivierungspolitischer Maßnahmen in Österreich im Rahmen der Sozialhilfereform und Einführung der Bedarfsorientierten Mindestsicherung (BMS) im Jahr 2010. Damit soll ein Beitrag zur Untersuchung der empirischen Effekte der normativen sowie praktischen Anforderungen von Aktivierung und deren institutioneller Praxis auf der Ebene von Subjekten geleistet werden. Die Überlegungen stehen im Spannungsfeld zwischen Subjektivierungsforschung, Armuts- und Governance-Forschung. In einem ersten Schritt werden wir auf die methodologischen Erfordernisse für die Untersuchung der Effekte und Aneignungsweisen sozialpolitischer Regulierung und dafür bisher vorliegende Studien eingehen, in einem zweiten die Spezifika der österreichischen Sozialhilfereform erläutern, um in einem dritten Schritt schließlich Forschungsdesign und Ergebnisse der diesem Beitrag zugrundeliegenden Studien ${ }^{1}$ zu den Aneignungsformen der Bedarfsorientierten Mindestsicherung in Österreich vorzustellen und damit eine Antwort auf die Frage nach der Aneignung aktivierungspolitischer Maßnahmen und das Gelingen oder Scheitern der Ziele des aktivierungspolitischen Paradigmas zu diskutieren.

\section{Zur Untersuchung des Verhältnisses sozialpolitischer Regulierung und Aneignungen von Subjekten}

Anders als die Form des Regierens selbst, haben die Effekte des aktivierungspolitischen Regimes auf die betroffenen langzeitarbeitslosen Subjekte - speziell in Österreich - bis dato wenig Aufmerksamkeit erfahren. Erst mit der Untersuchung der Aneignungsprozesse der diskursiven Anrufungen durch die ,regierten“ Subjekte kann jedoch etwas über die Wirkungsweise sozialpolitischer Reformen bzw. über die Wirkungsweise von Diskursen und Institutionen im Allgemeinen ausgesagt werden (vgl. auch Keller 2011). Entsprechen die Subjekte den systemischen Imperativen? Wie gehen Sie mit den diskursiven institutionellen Anrufungen um?

In der Klassiker-Studie Die Arbeitslosen von Marienthal konnten vier HaltungsTypen für den Umgang mit der Krisensituation „Arbeitslosigkeit“ beschrieben werden: „die innerlich Ungebrochenen“, ,die Resignierten“, „,die Verzweifelten“ und die

\footnotetext{
1 Der Beitrag greift auf Material der noch unveröffentlichten Habilitationsschrift der Autorin „Zwischen Autonomie und Heteronomie. Die Arbeitslosen der Aktivierungsgesellschaft“, zurück: Globisch 2016. Empirische Erhebungen und Auswertungen hierfür wurden in zwei Projekten an der Universität Innsbruck durchgeführt (,Armutsdynamiken in Tirol“ (gefördert vom TWF) sowie im Habilitationsnachwuchsförderungsprojekt „Handlungsautonomie und Krisenroutinen. Armutsdynamiken in Österreich“, gefördert von der Nachwuchsförderung der Universität Innsbruck). In den Projekten waren Jonathan Jancsary MA, Lukas Kerschbaumer, MA, Monika Liengitz, BA und Fabian Madlung, BABA als studentische Projektmitarbeiter beschäftigt und mit Interviewführungen, Transkriptionsarbeiten und der Erstellung von Auswertungsprotokollen betraut. Ausgewertet wurde in unterschiedlich zusammengesetzten Auswertungsteams mit den studentischen Mitarbeiter_innen sowie den hermeneutisch geschulten Fachkolleg_innen Dr. Andreas Hirseland, Prof. ${ }^{\text {in }}$ Dr. ${ }^{\text {in }}$ Lisa Pfahl und Dr. Boris Traue, denen an dieser Stelle herzlich für Ihre Zeit und Muße gedankt sei.
} 
„,verwahrlost Apathischen“. Die Vorstellung der Veränderbarkeit von Zukunft und des Entwurfs von Planungshorizonten wurde dabei als entscheidendes Kriterium identifiziert, um den ersten Typus der ,innerlich Ungebrochenen“ von den anderen drei Typen zu unterscheiden (vgl. Jahoda et al. 1975; zuerst 1933).

Die Studien zu Armut und Arbeitslosigkeit in den 1990er-Jahren in Deutschland zeigen, dass sich weitere Haltungs- und Handlungsformen im Umgang mit der Arbeitslosigkeit entwickelt haben. So werden in der Bremer Armutsdynamiken-Studie fünf Typen des Umgangs mit Sozialhilfe unterschieden: „Opfer“, „Problemverwalter", ,pragmatische Gestalter“, ,aktive Gestalter" sowie „strategische Nutzer“, wobei den ,aktiven Gestaltern“ im qualitativen Sample von 31 Fällen die größte Ausprägung zugeordnet werden konnte (vgl. Leibfried et al. 1995). Im Vergleich zu den Arbeitslosen von Marienthal wurden hier weit mehr Handlungsmächtigkeit und aktive Gestaltung der eigenen Situation beobachtet. Den Zielen des Aktivierungsparadigmas zufolge, das sich in Deutschland maßgeblich mit den Hartz-Reformen (das Erste bis Vierte Gesetz für moderne Dienstleistungen am Arbeitsmarkt), in Österreich mit der Änderung des Arbeitslosenversicherungsgesetzes und der Reform der Sozialhilfe 2010 realisierte, sollten die sozialpolitischen Maßnahmen weiteren Einfluss auf die Aktivierung von Arbeitslosen haben. Ob dies tatsächlich der Fall ist und ob die Aktivierung gemäß den sozialpolitischen Zielen erfolgt, ist eine empirische Frage, die in diesem Beitrag verhandelt wird.

Was die verschiedenen in Deutschland erhobenen Querschnitts- und Längsschnittstudien vereint (Ludwig-Mayerhofer et al. 2009; Dörre et al. 2008; Hirseland und Ramos Lobato 2010; Weißmann 2016), ist die Beobachtung der zentralen Bedeutung von Erwerbsarbeit als normativer Referenzrahmen einerseits, die der hohen Aktivität der arbeitslosen TransferleistungsempfängerInnen und ihre eigensinnigen Aneignungs- und Inklusionsleistungen, die jedoch nicht immer Aktivierung im Sinne der sozialpolitischen Ziele (vgl. insbes. Hirseland und Ramos Lobato 2010), sondern alternative Aktivität in verschiedenen sozialen Feldern und damit verbundener Anerkennungsaufbau bedeuten kann (vgl. insbes. Weißmann 2016), andererseits.

Während Weißmanns Studie dabei insbesondere auf die Zugehörigkeitskonstruktionen als Inklusionsleistungen fokussiert, stellen die Studien von Mayerhofer, Sondermann und Behrend die Autonomieverluste von arbeitslosen TransferleistungsempfängerInnen heraus. In unseren Studien zeigt sich, dass beide Aspekte miteinander verzahnt sind: Spezifische Zugehörigkeitskonstruktionen sind auch Folgen von Autonomieverlusten, die beispielsweise xenophobe Abwertungsmechanismen nach sich ziehen, aber auch Zughörigkeitskonstruktionen zur gesellschaftlichen Mitte, die im Gegensatz zu den eigenen Narrationen prekärer Alltagslagen stehen, können Folge von Autonomieverlusten in der Langzeitarbeitslosigkeit sein.

Wie die vorhandenen Studien augenscheinlich zeigen, sind die Aneignungsprozesse zwar durch (makro-)strukturelle diskursive, institutionelle Rahmenbedingungen (Arbeitsmarkt, Gesetze und rechtliche Vereinbarungen, Wohlfahrtsinstitutionen, politische Programme) bedingt, werden aber individuell vor dem Hintergrund der jeweiligen biografischen Erfahrungen umgesetzt. Daher ist es notwendig, einerseits diese strukturellen Kontexte zu rekonstruieren und andererseits fallbezogene Rekonstruktionen typischer Handlungsprozesse durchzuführen. Da derzeit Langzeitarbeitslose aktivierungspolitisch regiert werden, ist dies das sozialpolitische Setting, 
das einen zentralen Kontext für die subjektiven Bearbeitungen darstellt. Die dazu notwendige methodische Systematik muss das Verhältnis zwischen den rechtlichen, politischen und medialen Diskursen, den institutionenspezifischen Logiken sowie den subjektiven, biografisch und milieuhaft eingebetteten Deutungen in den Blick nehmen können.

Eine Möglichkeit, das zu realisieren, besteht in der Verknüpfung von diskursanalytischen und ethnografischen mit biografietheoretischen Zugängen. An der methodischen Verknüpfung zwischen Diskurs- und Biografieforschung sind in den letzten zehn Jahren einige Forschungsprogramme unter dem Stichwort „Subjektivierungsforschung" entstanden (vgl. etwa Keller et al. 2012; Pfahl et al. 2014; Bosančić 2014, 2016; Geimer 2014), für den Gegenstandsbereich der Armutsforschung finden sich hier allerdings wenige systematische Studien. Die qualitative Datenlage, die über das Verhältnis institutioneller Steuerung, diskursiver Anrufung und individueller Aneignung aufklären könnte, ist also stark unterrepräsentiert. Dieser Mangel an qualitativen Studien trifft in Zusammenhang mit der Reform der Sozialgesetzgebung mit Einführung der Bedarfsorientierten Mindestsicherung auf wachsenden Bedarf - bislang existiert bis auf wenige Ausnahmen (etwa L\&R Sozialforschung 2011) kaum Forschung zu den Auswirkungen und subjektiven Aneignungsformen der BMS: Nicht nur angesichts der Vielzahl dichter Studien und Erkenntnisse etwa zur äquivalenten sozialpolitischen Reform in Deutschland (SGB II bzw. Hartz IV) (z. B. Hirseland et al. 2007; Hirseland und Ramos Lobato 2010; Ludwig-Mayerhofer et al. 2009; Grimm et al. 2013; Weißmann und Sammet 2010; Weißmann 2016) tut sich in Österreich hier eine eigentümliche Leerstelle auf. Dies umso mehr, als im Kontext der „Flüchtlingskrise“, der allgemein prekären Wirtschaftslage sowie der kontinuierlichen Erstarkung rechtspopulistischer Diskurse und Politiken die Mindestsicherung 2016 erneut in den Mittelpunkt einer revisionistischen, neoliberalen Agenda gerückt ist (vgl. etwa derStandard.at 2016), deren Spuren sich in der seit Beginn des Jahres 2017 in mehreren Bundesländern gültigen Novelle des Mindestsicherungsgesetzes abzeichnen: eine Deckelung der Mindestsicherung sowie eine Einschränkung der Anspruchsvoraussetzungen für Personen, die in den vergangenen sechs Jahren weniger als fünf Jahre ihren Hauptwohnsitz bzw. rechtmäßigen Aufenthalt in Österreich hatten.

\section{Die österreichische Sozialhilfereform}

In Österreich als - gemäß der Typologie Esping-Andersons - konservativ eingestufter Wohlfahrtsstaat waren die Sozialhilfereform und Einführung der Bedarfsorientierten Mindestsicherung 2010 die konsequente Fortführung einer bereits in den 1970-Jahren vorbereiteten, in den 1980ern aktualisierten, seit Mitte der 1990er konkretisierten und in den Nuller-Jahren endgültig realisierten aktivierenden Arbeitsmarktpolitik (vgl. Atzmüller et al. 2012).

Bei der BMS handelt es sich, anders als die in Deutschland unter dem Stichwort „Hartz IV“ bekannt gewordene Zusammenlegung von Sozialleistungen, um eine „reine“ Reform der Sozialhilfe. Das heißt, dass es damit auch nicht wie in Deutschland zu Statusabwertungen durch die Zusammenlegung von unterschied- 
lichen Statusgruppen gekommen ist. Dies mag vielleicht auch erklären, dass in unserem Sample die Stigmatisierungserfahrungen mit der Bezeichnung ,MindestsicherungsempfängerIn" in der Selbstwahrnehmung weniger stark ausgeprägt sind als bei den „Hartz-IVlerInnen“ und in den Erzählungen oftmals nicht zwischen „Mindestsicherung“ und „Sozialhilfe“ als konsekutiven Systemen unterschieden wird. Im nach wie vor dreiteiligen österreichischen Leistungssystem nimmt die BMS die Stelle des letzten Netzes ein, so wie vormals die Sozialhilfe. Der wesentliche Unterschied besteht darin, dass die Mindestsicherung im Gegensatz zur den Bundesländern überlassenen Sozialhilfe einen bundesweit einheitlichen Mindestsatz vorsieht, die Organisation aller Transferleistungen unter einem Dach („One-Stop-Shop“), dem Arbeitsmarktservice (AMS), vorsieht sowie von einer ,aktiven Arbeitsmarktpolitik“ mit diversen Arbeitsanreizen begleitet wird und als nationale Umsetzung der europäischen Beschäftigungsstrategie betrachtet werden kann. Mit der Reform wurde auch der Berufsschutz gelockert und die damit verbundenen Sanktionierungsmöglichkeiten bei Nicht-Arbeitswilligkeit verschärft. Die Verschärfung der Zumutbarkeitskriterien und damit die Erweiterung der Bestimmung von Arbeitswilligkeit wurde jedoch bereits in der Novelle des Arbeitslosenversicherungsgesetzes aktivierungspolitisch verschärft (vgl. Atzmüller 2009). Nach wie vor wird die Mindestsicherung zwar in Landesgesetzen organisiert, dabei aber durch eine Vereinbarung mit dem Bund nach unten gedeckelt und grob geregelt. In allen drei Gliedern - den versicherungsbasierten Leistungen Arbeitslosengeld und Notstandshilfe sowie der Mindestsicherung wird Arbeitswilligkeit unter Sanktionsandrohung vorausgesetzt. Die politische Debatte während der Einführung der BMS wurde in starker Referenz auf den deutschen Hartz-IV-Diskurs, dabei aber vor allem mit Fokus auf die Vermeidung befürchteter passivierender Effekte und Missbrauch von Sozialleistungen geführt und nicht auf die Sicherung des Existenzminimums. Gerade diese diskursive Wendung macht deutlich, dass die österreichische Debatte weniger auf Armutsvermeidung, sondern vielmehr auf Arbeitsaktivierung abstellt.

Die zur Verfügung gestellte Leistung fällt demnach auch sehr knapp aus, 2016 liegt sie für Alleinstehende bei 837,76€ und damit unter der Armutsgefährdungsschwelle, die in Österreich laut EU-SILC-Daten (2014) bei $1161 €$ angesiedelt ist. Zwar ist erklärte Absicht, ,eine angemessene soziale und kulturelle Teilhabe“ (§ 7) zu ermöglichen, kulturelle Güter oder soziale Zusammenkünfte sind in den Mitteln jedoch nur marginal kalkuliert.

\section{Methodologie und Sample}

Um die Frage nach den Aneignungsprozessen der sozialpolitischen Reformen zu beantworten, stehen im Vordergrund der folgenden Überlegungen nicht die eingangs bereits diskutierten aktivierungspolitischen Maßnahmen, sondern die Typen der Handlungsaktivierung. Wie werden die HilfeempfängerInnen im Rahmen der sozialpolitischen Reformen aktiviert? Geschieht dies gemäß den sozialpolitischen Zielen der Employability oder anders? An diesen Fragen setzt unsere empirische Forschung an. 
In Anlehnung an die Tradition der Erforschung von „Armutsdynamiken“ (vgl. etwa Leibfried et al. 1995) wurden daher einerseits rekonstruktive biografietheoretische Methoden verwendet, diese jedoch mit diskursanalytischen Untersuchungen verknüpft, um sowohl die dynamischen Prozesse und Pfadabhängigkeiten zu untersuchen, die zum Eintritt in die Hilfebedürftigkeit geführt haben, als auch die Subjektivierungs- und Aktivierungsprozesse und ihr Verhältnis zu den institutionellen und diskursiven Kontexten in den Blick zu bekommen. Den Ergebnissen der Bremer Armutsdynamiken-Studie folgend gingen wir davon aus, dass die subjektiven Aneignungen zwar durch makrostrukturelle Rahmenbedingungen (Arbeitsmarkt, Gesetze, Wohlfahrtsinstitutionen, politische Diskurse) präfiguriert sind, aber individuell vor dem Hintergrund der jeweiligen biografischen Erfahrungen umgesetzt werden, d. h. die arbeitslosen TransferleistungsempfängerInnen nicht nur Opfer und Erleidende, sondern auch Handelnde sind (vgl. Leibfried et al. 1995, S. $176 \mathrm{ff}$.) und empirisch zu untersuchen ist, ob der Transferleistungsbezug dabei als Hilfe und/oder Kontrolle erlebt wird und wie die institutionellen Diskurse und Semantiken aufgegriffen werden.

Mit Hilfe von Grounded-Theory-angeleiteten Analysen (vgl. Glaser und Strauss 1967; Przyborski und Wohlrab-Sahr 2010) der Rechtstexte, politischen Programmatiken und Stellungnahmen zur Mindestsicherung sowie 18 halbnarrativen ExpertInneninterviews mit BeraterInnen und VermittlerInnen wurden die institutionellen und diskursiven Rahmenbedingungen rekonstruiert. Dafür wurden die $\S 15 \mathrm{a}-\mathrm{Bun}$ desvereinbarung und ausgewählte Ländergesetze ausgewertet. Die Anrufung der Subjekte als aktivierbare arbeitsfähige und für ihre Lage und deren Überwindung selbstverantwortliche Subjekte, die sich für den Erhalt sozialstaatlicher Leistungen bewähren und unbedingt arbeitswillig zeigen müssen, kann als gemeinsamer Nenner der Anrufungen vonseiten der institutionellen VertreterInnen zusammengefasst werden. Die BeraterInnen variieren abhängig von ihrem beruflichen Selbstverständnis zwischen SozialarbeiterInnen, TrainerInnen und Verwaltungsangestellten, reichen in ihren Anrufungen und Methoden der Bearbeitung der Subjekte von TrainerInnen mit Erziehungscharakter zu proaktiv kontrollierenden VerwaltungsbeamtInnen. Der „Missbrauchs“- und ,soziale Hängematten“- bzw. „Faulheits“-Diskurs sowie der „Bewährungs“-Diskurs sind sowohl bei den institutionellen VertreterInnen als auch im politischen Diskurs präsent. Im politischen Diskurs wird von verschiedenen Sprechorten auf den „Hängematten“- und „Missbrauchs“-Diskurs Bezug genommen und die BMS entweder dahingehend verteidigt, keine soziale Hängematte zu ermöglichen, oder aber dafür kritisiert, ebendies zu tun.

Im Zeitraum zwischen 2013 und 2015 wurden im Rahmen unserer Studien 26 eineinhalb- bis dreistündige narrativ-biografische Interviews mit EmpfängerInnen der Bedarfsorientierten Mindestsicherung in städtischen und ländlichen Regionen Österreichs geführt. Dabei fanden sich Personen unterschiedlicher Bildungsmilieus zwischen 21 und 60 Jahren, mit und ohne Migrationshintergrund (Deutschland, Sudan, Ägypten, Tschetschenien, Türkei, Bulgarien), mit und ohne Betreuungsverpflichtungen (alleinerziehende Männer und Frauen mit gesunden und behinderten Kindern, Paare mit Kindern), Teil- und VollbezieherInnen im Sample. Auswahlkriterium war, dass sie zum Interviewzeitpunkt BezieherInnen der Bedarfsorientierten Mindestsicherung waren. Ausgewertet wurden die Interviews mittels biografie- 
theoretisch angeleiteter, sequenzanalytischer Verfahren in der Tradition Oevermanns (vgl. Oevermann et al. 1979; Oevermann 2000), allerdings ohne seine ontologischen Annahmen (vgl. die Diskussion in Bezug auf ihre Bedeutung für empirische Auswertungen: Weisenbacher 1993; Holz 2001; Globisch 2013). Dabei wurde in einem ersten Schritt die Abfolge lebensgeschichtlicher Ereignisse interpretiert, um in einem zweiten Schritt die narrative Aneignung des erlebten Lebens durch die Subjekte zu rekonstruieren. Die Narrationen werden dabei als Stegreiferzählungen im Sinne Fritz Schützes (vgl. Schütze 1984) mitsamt den dafür typischen Erzählzwängen verstanden. Wir verstehen dabei die biografischen Erzählungen nicht als reine Spiegelung von Diskursen, sondern die institutionellen diskursiven Kontexte werden als „Bewährungs- und Zumutungskontexte, aber auch als Legitimationsressourcen“ (Pfahl et al. 2015, S. 101) aufgefasst, vor deren Hintergrund sich die Logiken biografischen Handelns entfalten.

\section{Idealtypen der Handlungsaktivierung}

Im Zuge unserer empirischen Auswertung erschloss sich ein biaxialer Raum, entlang dessen zwei Achsen x) Systemimperative - Eigensinn und y) Ermächtigung - Entmächtigung sich die einzelnen Fälle gruppieren und zu Idealtypen zusammenfassen ließen (Abb. 1). Das Achsenkreuz (fett umrandet) konstituiert hier vier Quadranten, in denen sich Subjekte entlang zweier, von vier Polen begrenzter Spektren verorten lassen (fett): Systemimperative leiten Subjekte an, sich entsprechend normativer gesellschaftlicher Vorgaben zu orientieren und $\mathrm{zu}$ vergesellschaften - diese

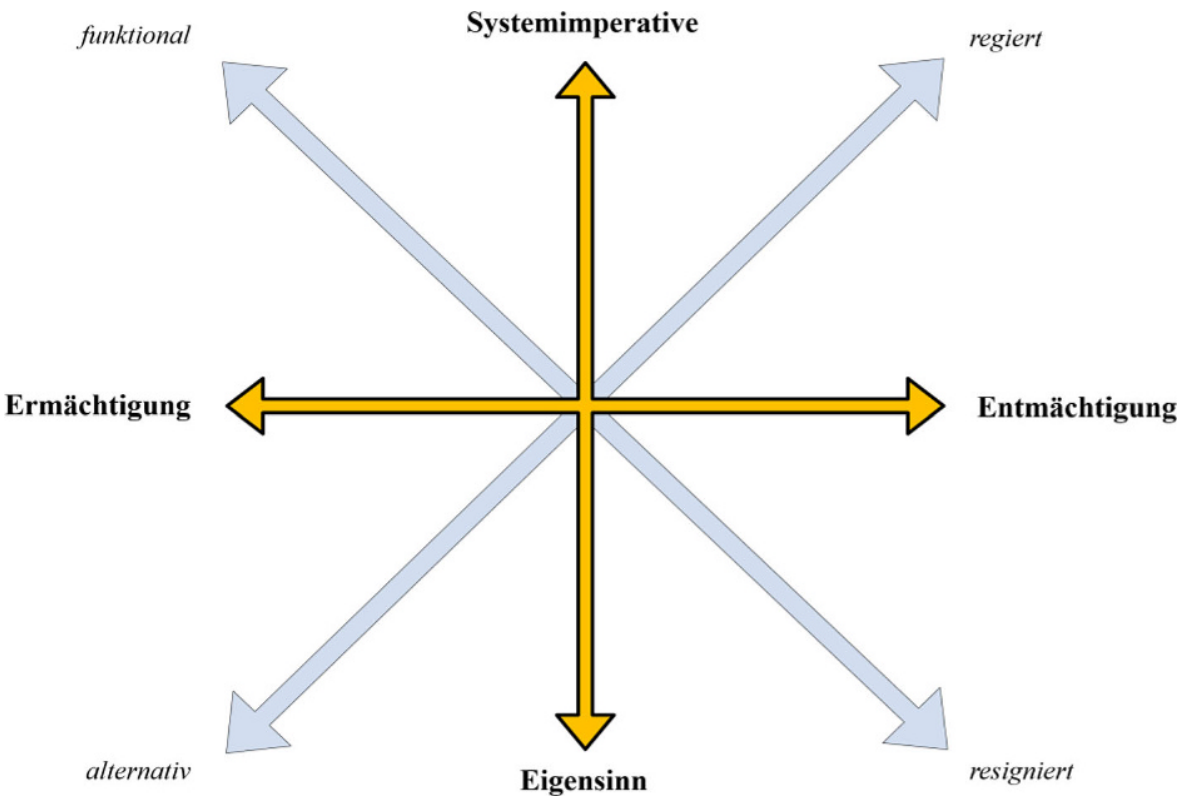

Abb. 1 Haltungs- und Handlungsaktivierungstypen 
werden artikuliert, vertreten und durchgesetzt von den Agenturen und Einrichtungen des Staates (und Marktes); dagegen reflektiert die Betonung des subjektiven milieubedingten Sinnhorizonts, also die Dominanz des Eigensinnes gegenüber den Obligationen der Arbeitsgesellschaft und ihrer Institutionen, die graduelle Autonomie bestimmter Subjekte gegenüber den Forderungen, aber auch Versprechungen des Staates. Zwischen den beiden Polen besteht ein Kontinuum von Positionierungen, die Subjekte im Verhältnis zur staatlichen bzw. institutionellen Regulierung einnehmen können. Dasselbe gilt für die horizontale Achse, die ein Spektrum zwischen den Handlungsformen - im interdependenten Wechselspiel staatlichen Regierens und individuellen Reagierens - Ermächtigung und Entmächtigung beschreiben: Orthogonal zur vertikalen „Konformitäts“-Achse beschreibt die „Empowerment“-Achse die Gestaltungs- und Handlungskompetenz von Subjekten. Sie umspannt mögliche Varianten zwischen (autonomer) Ermächtigung und (heteronomer) Entmächtigung. Zwischen und im Verhältnis dieser vier komplementären Konstituenten eines sozialen Raumes positionieren sich (oder werden positioniert) Subjekte, die Prekarität in verschiedenen Formen gemein haben. Aus der Relationierung in diesem Raum ergeben sich, je nach Ort, den ein Subjekt einnimmt, schließlich bestimmte „Haltungs““Typen bzw. Typiken von Verhältnissen zur Erwerbsarbeitsgesellschaft (kursiv). Wer im Sinne der Systemlogik ermächtigt wird, wird von uns als funktional bestimmt. Wer kein Empowerment, d. h. volle Marktintegration erfährt, aber innerhalb der Institutionen prozessiert bleibt, gilt uns als regiert. Als resigniert begreifen wir diejenigen Personen, die bei individuell entmächtigter Lethargie vom System und seinen Agenturen nicht mehr als aktivierbare, insofern integrierbare Subjekte adressiert werden. Schließlich beobachten wir alternative Entwürfe, die abseits der normativen Systemimperative ein graduell selbstbestimmtes Leben bzw. selbstbestimmte Lebensbereiche ermöglichen. Insgesamt zeigt sich also ein schematischer Raum, in dem „Aktivierung“ nicht allein institutionelle Agenda, sondern auch subjektive, nicht notwendig systemkonforme Aneignung bedeuten kann - und, umgekehrt, in dem auch passivierende Effekte innerhalb institutioneller Strukturen beobachtbar sein können:

Aus dem zwischen den Achsen aufgespannten Raum, der die Fälle spektral ordnet, ergeben sich vier Extrempositionen an den Ecken des Quadrates, dessen Mittelachsen die Hauptdichotomien bilden. Sie kondensieren wir zu Idealtypen, denen die Haltungsadjektive zugeordnet werden:

„Die Funktionalen“, in der linken oberen Ecke, sind das deklarierte Desiderat des sozialpolitischen Systems und seiner intrinsischen Logik. Dieser Idealtypus beschreibt jene Fälle, in denen sich die Ansprüche der aktivierenden Arbeitsmarktpolitik an ein gelungenes Case-Management am weitesten mit dem konkreten Verlauf des Falles decken; das Subjekt wurde innersystemisch zum funktionierenden Erwerbsarbeitssubjekt aktiviert. Die Transferleistung und ihre Begleitmaßnahmen sind hier temporäre Relais und transitorische Phasen, die die betroffenen Subjekte in den oder zurück in den regulären Arbeitsmarkt prozessieren. Insofern bleibt der Typ in der oberen Hemisphäre des Kreuzes, sein Subjekt verlässt - für den für uns untersuchungsrelevanten Bereich - nicht die Systemebene und ihre normativen Imperative, sehr wohl aber den Bereich der heteronomen Krise. Dieser Typus realisiert sich empirisch am seltensten. In unserem Sample finden sich hier insbesondere vom System 
im Vorhinein besonderte Subjekte - etwa entlang der Kategorie „behindert“ -, deren Streben nach gesellschaftlicher Anerkennung intrinsische Motivation und für die der Weg in die normativ vorgesehene Sphäre des Normalerwerbs ein Lösungsversprechen ist, oder aber Gebildete, die ihre Ressourcen, d.h. vor allem ihr kulturelles Kapital für die (Wieder)Befähigung zur Erwerbsarbeit nutzen können.

Als Referenzfall steht hier eine „lernbehinderte“ Arbeitslose, die zum Zeitpunkt des Interviews seit einem Jahr als ,arbeitsfähig“ in Teilzeit eingestuft wurde. Frau Weiß wurde 1975 in einer österreichischen Großstadt als Tochter eines Tischlers und einer gelernten Kürschnerin geboren ${ }^{2}$ und wuchs zusammen mit einem jüngeren Bruder, der Autist ist, auf. Ein Badeunfall im Kindesalter, bei dem die Befragte eine Hirnschädigung erlitt, prägt ihre Biografie. Diese ist gezeichnet durch physische und symbolische Gewalterfahrungen und den auf Testverfahren basierenden Klassifikationspraxen (vgl. hierzu auch Schreiber-Barsch und Pfahl 2014) einer Gesellschaft, die Personen in „Normale“ und „Behinderte“ bzw. ,Deviante“, „Fähige“ und „Nicht-Fähige“ einteilt und daran spezifische Rechte knüpft (z. B. den Besuch von bestimmten Schulen, Ausbildungen etc.). Die Erfahrung dieser Klassifikationspraxen und daran geknüpfte Konsequenzen des „Nicht-Dürfens“ und „Nicht-Könnens“ prägt die gesamte Erzählung. Die durch den Unfall ausgelöste „Lernschwäche“ schlägt sich in einem $50 \%$-Behindertenstatus nieder, der von der Befragten als unsachgemäße und heteronomiefördernde Einstufung und Abwertung wahrgenommen wird (,,weil, nur weil ich diesen Unfall gehabt hab', haben sie mich als fünfzig Prozent * Behinderung geschrieben “), gegen die sie Zeit ihres Lebens ankämpft. Sie selbst beschreibt sich als „langsamer lernend“. Nach dem Abschluss der polytechnischen Integrationsklasse befindet sich Frau Weiß sieben Jahre in einer Beschäftigungstherapie in zuerst voll-, dann teilbetreuten Wohnarrangements (WG, teilbetreutes Wohnen), bis sie schließlich mit 27 Jahren in eine Gemeindewohnung ziehen kann. Es gelingt ihr, die Beschäftigungstherapie in einer Küchen-/Haushaltslehranstalt abzuschließen und 2014 eine dreijährige Teilausbildung zur Kindergartenassistentin, ihrem Wunschberuf, zu absolvieren. Seither ist sie arbeitslos, bezieht die BMS und ist Teilnehmerin eines Arbeitsvermittlungsprogramms bei einem ausgelagerten Personalserviceunternehmen des AMS.

Jeder Schritt hin zu weniger Betreuung und Angewiesenheit auf Transferleistungen bedeutet für Frau Weiß einen Autonomiegewinn: zuerst die Beendigung der Betreuung und Besachwaltung, dann der Umzug von der betreuten Wohnform in die eigene Gemeindewohnung und zuletzt die Einstufung vom Status „arbeitsunfähig “ zum Status „arbeitsfähig“. Für sich selbst entscheiden zu können und ihr Leben selbstbestimmt führen zu können, ist für unsere Befragte zentral mit einem eigenen Einkommen jenseits von Transferleistungen verbunden. Ihr größtes Ziel ist daher, „mein eigenes Geld verdienen“:

Und ich hab' immer gesagt ich, ich möchte nicht mein ganzes Leben lang von Sozialamt leben, ich möcht' * mein eigenes Geld verdienen. [. . .] seit zwei Jahren * ähm * bin ich nicht mehr betreut. [. . .] Und auch nicht mehr

\footnotetext{
${ }^{2}$ Die Eltern sind mittlerweile geschieden. Die Befragte pflegt regelmäßigen Kontakt mit ihrer Mutter und ihrem Bruder, der in einem betreuten Wohnarrangement lebt. Zum Vater besteht kaum Kontakt.
} 
besachwaltet, also * ich habe das * geschafft, dass ich da wegkomm'. [. . .] Weil ich gefunden hab', ich weiß nicht, ich, * die haben mir alles weggenommen, Kontokarte, Dokumente und so. [. . .] Und ich hab' das dann irgendwie nicht, * also von meiner Sicht war das nicht in Ordnung, weil sie haben mir keine Chance gegeben, um zu beweisen, dass ich das auch selber kann, also ...

An Frau Weiß wird deutlich, dass „,eigenes Geld“ aus abhängiger Lohnarbeit solch einen hohen Stellenwert hat, weil es mit einem Übertritt von den „Integrationsleuten“, wie sie ihre Zugehörigkeit beschreibt, zu den „normalen Leuten “ verbunden ist und das Leben in Besachwaltung und Beschäftigungstherapie als entmündigend erfahren wurde (die haben mir alles weggenommen; weil sie haben mir keine Chance gegeben, um zu beweisen, dass ich das auch selber kann). Obwohl sie sich von den BeraterInnen des AMS erniedrigt und „herumgeschickt“ fühlt sowie in passungenaue Kurse (fortgeschrittener Englischkurs ohne englische Basiskenntnisse) vermittelt wird, ist sie bereit, jede Arbeit anzunehmen, die ihr mehr Einkommen verspricht als die derzeitige Transferleistung und ihr Aussicht auf die Zugehörigkeit zu den „,normalen Leuten“ ermöglicht. Neben dem Wunsch, damit den Stigmatisierungsprozessen zu entkommen, erhofft sich Frau Weiß davon eine Normalisierung ihrer finanziellen Situation, nämlich Geld beiseitelegen zu können, um kaputtgegangene Dinge ersetzen oder sich auch mal einen Urlaub leisten zu können.

Durch Vollzeitarbeitsfähigkeit und -tätigkeit ,,auf eigenen Beinen stehen “, ist für sie das große Ideal. Dieses Ziel bleibt ihr jedoch verwehrt.

Weil eben drinnen steht, ich soll nichts Schweres heben und, * und dass ich, * ich weiß nicht, ich hab' das irgendwie auch ein bisschen beleidigend empfunden, dass ich für den ganzen Tag nicht zu gebrauchen bin und so. [...] Und ich hab' gehört, aber, wenn ich jetzt Teilzeit arbeiten geh', * hm bekomm' ich ja wieder was von Sozialamt dazu. \#JA\# Und das wollt' ich eben vermeiden.

Der Fall zeigt, dass die aktivierenden Maßnahmen, die von der Möglichkeit und expliziten Forderung einer individuellen Überwindung von Defiziten ausgehen, trotz eigener Bemühungen, unbedingter Arbeitswilligkeit und Aktivität ins Leere laufen, da für Frau Weiß' Bedürfnisse am Arbeitsmarkt keine passenden Stellen verfügbar sind bzw. bereitgestellt werden. Trotzdem wird ihr durch die aktivierungspolitischen Maßnahmen vermittelt, es handle sich dabei um ein individuelles „Defizit“. Die Jobsuche stellt sich daher trotz der als Aufstieg erlebten Einstufung als ,,arbeitsfähig““ deprimierend dar und hinterlässt sie wiederum mit dem Gefühl: „Ich hab’ (bzw. kann, Anm.) gar nichts":

[. . . ] jetzt\# freu' mich halt darüber, dass ich arbeitsfähig bin, aber ich freu' mich nicht darüber, dass ich keinen Job find'. (LACHT) . . . Das ist der Nachteil daran. . . . Und es ist auch nach einer Zeit dann anstrengend, * . . . weil man dann wirklich genau schauen muss: Bin ich dafür geeignet, bin ich dafür nicht geeignet, also ...** Zum Beispiel als Regalbetreuer, es gibt auch Lagerarbeiter, da brauchst einen * Staplerschein. [. . .] Und den hab' ich nicht. . . . Ich hab' auch keinen Führerschein. Ich hab’ gar nichts. 
Der zweite Idealtypus der „Regierten“, in der rechten oberen Ecke, repräsentiert jene häufigeren Fälle, in denen Subjekte zwar nicht resigniert aus dem System ausscheiden, aber das Ermächtigungs- und Funktionalitätskriterium von Typ 1 nicht erfüllen. Sie bleiben systemisch eingebettet, werden dabei aber nicht ermächtigt - weder zu erwerbsarbeitskompatiblen noch zu lebensweltlich speziell kreativen Subjekten, die Sinn- und/oder Erwerbsquellen jenseits des formalen Arbeitsmarktes suchen und finden. In diesem Quadranten des Schemas begreifen wir Subjekte als ,aktiviert“, allerdings nicht im Sinne des erwerbsarbeitszentrierten Paradigmas, sondern vielmehr darin, als sozial- und arbeitsmarktpolitisch prozessierte Subjekte zu bestehen und aktiv als verwaltete BürgerInnen an ihrer Prozessierung teilzuhaben.

Beispielhaft lässt sich hier Herr Heim anführen, der 1975 in eine Broken-homeFamilie mit niedrigem Bildungsstatus geboren wurde und eine Kindheit geprägt von Gewalt und Alkoholismus erlebte. Bis heute lebt er in einer industriell geprägten Gegend im Westen Österreichs. Seine Biografie zeichnet sich nach der Absolvierung der Pflichtschule durch eine lange Periode apathischer Nicht-Teilnahme am Erwerbsarbeitsprozess aus: 1990 zieht er nach Gewalterfahrungen durch seinen Stiefvater zu den Großeltern. Ab hier beginnt eine von vielfältigen Drogen und Hafterfahrungen geprägte Zeit, in der er nie ein Einkommen oder Transferleistungen bezogen hat, sondern nur von der marginalen Unterstützung der (selbst prekär lebenden) Mutter sein Auskommen finden muss. Diese Phase endet erst 17 Jahre später mit Kennenlernen eines über eine Freundin vermittelten „Lebensberaters“, durch den er erst von der Möglichkeit der damals noch bestehenden Sozialhilfe erfährt. Mit dieser Begegnung im Jahr 2007 beginnt eine Öffnung und ein ,therapeutisierter“ Lebenswandel, begleitet durch Sozialhilfe, die ab 2011 in die BMS überführt wird. In verschiedenen therapeutischen und strukturierenden Maßnahmen, von der psychiatrischen Betreuung in der Tagesklinik über alltagsstrukturierende Beschäftigungsinitiativen bis hin zur Arbeit in einem Berufstrainingszentrum für Menschen mit psychischen Problemen, beginnt Herr Heim sich ein bürgerliches Erwerbsarbeitsleben vorstellen zu können. Allerdings gelingt ihm die Konsolidierung nur bedingt. Er verbleibt jahrelang in einem wechselhaften Modus von Abbruch und Neuanfang. Die von der BMS geforderte Arbeitswilligkeit erfüllt er in den verschiedenen Maßnahmen, wird aber nicht befähigt, sie auf die Etablierung eines selbständigen, existenzsichernden Erwerbsleben zu richten:

Uuund äääh *4* JA *, daaann bin ich, hab' ich das WIEder abgebrochen, dann hab' ich GANZ viel stationäre Aufenthalte gehabt, und hab' auch Mindestsicherung bezogen, Gott sei Dank, ge, uuund aaah, aaah dann * bin ich das erste Mal in die Beschäftigungsinitative * gekommen, ich weiß nicht, ob ihr sowas kennt, das ist eine Tagesstruktur, * also wo man hingeht, dass man was am Tag tut, und da gibt's eine HALBtagesstruktur, und da haben wir zuerst gemeint, wir fangen mit der Halbtagesstruktur, einmal wieder langsam (HOLT LUFT) zurückkommen, ins Leben, das hab' ich dann * damals NICHT derPACKT, also das ist über MOnate gegangen, aber ich hab's, ich bin's oft nicht aufderstanden, [. . .] Uuund ääähm dann ist daaas WIEder, dann hab' ich gedacht, „Ja super, was tu' ich denn jetzt, steh' ich WIEder d//“", also ich $\mathrm{h} /$, die, da ist mir dann schon lANge der Knopf aufgegangen, dass ich immer 
was tun MUSS. * Ge. Dass es so nicht w//, also und Ding, uuund dann hab' ich die Frau Doktor von der Tagesklinik angerufen, ob ich, ob iiich, ob ich noch einmal in die Tagesklinik kommen kann [. . .], und dann hab' ich das ZWEIte Mal die Tagesklinik, äh, besuchen dürfen. * (SCHLUCKT) Es hat mir dann WIEder wahnsinnig viel geholfen, obwoh//, uuund daaann äääh * nach der Tagesklinik, äh, bin ich dann in die BEschäftigungsinitiative gegangen, WIEDER, aber in die GANZtagesstruktur, weil's in der Tagesklinik immer ein bisschen BESser geworden ist, eigentlich, ge. Und das hab' ich dann ein Jahr durchgezogen, diiie Beschäftigungsinitiative, (HOLT LUFT) uuund dann die hat letzten AuGUST äääh aufgehört, uuund seit März bin jetzt da [in einem Berufstrainingszentrum, Anm.].

An der Interviewsequenz wird die dankbare Angewiesenheit (,,Gott [und an anderer Stelle dem Therapeuten, Anm.] sei Dank“; „,die Tagesklinik, äh, besuchen dürfen") auf Assistenz und Führung als Form institutioneller Regierung und internalisierte normative Aufforderung zur Beschäftigung und Etablierung eines ,,strukturierten“ Tagesablaufs deutlich, die in der Semantik der aktivierenden Sozialpolitik die intrinsische Logik darstellt, wenn auch mit dem nominellen Ziel der Arbeitsmarktintegration. Unter sanfter Anleitung eines Apparates aus ExpertInnen und Case-ManagerInnen ist Herrn Heim ,,der Knopf aufgegangen, dass ich immer was tun MUSS “.

Als dauerhaft prozessierte, sozialstaatlich disziplinierte und regierte „Sozialverwaltungssubjekte“ befinden sich die Betroffenen typischerweise für längere Zeit in der Sozialverwaltung, changieren zwischen Maßnahmen, Vermittlungsversuchen und Transferleistungen, können sich aber nicht in der Sphäre des regulären Arbeitsmarktes konsolidieren. Stark repräsentiert in diesem Quadranten finden wir normativ am normalen Arbeitsmarkt orientierte und mit ihm identifizierte Subjekte, die aber mit Bildungs- oder Ressourcendefiziten nicht in der Lage sind, ihr Streben zu realisieren.

Neben „geläuterten“, vormals deaktivierten Subjekten wie Herrn Heim finden sich in diesem Typus häufig auch etwa (jüngere) Alleinerziehende, die den Flexibilitätsansprüchen des Arbeitsmarktes nicht entsprechen können und während vieler gescheiterter Vermittlungsversuche im Sozialverwaltungssystem verbleiben. Wie an dem beschriebenen Fall beispielhaft dargelegt, sind die Subjekte hier nach wie vor ,aktiv“, werden aber nicht zu funktionierenden Erwerbsarbeitssubjekten im Sinne des Systemimperativs ,aktiviert“.

Idealtypus 3 in der rechten unteren Ecke, ,, die Resignierten “ bezeichnend, ist am weitesten entfernt vom (sozialpolitischen) Ideal der aktivierten, regulär Berufstätigen (Typ 1). In der unteren Hemisphäre des Kreuzes angesiedelt, sind entsprechende Subjekte weitestgehend entkoppelt von den Verfahren der Sozialverwaltung; zwar sind sie de jure die logische „Zielgruppe“ der Sozialverwaltung und als solche auch registriert und anerkannt, haben aber de facto keinen Status als ,aussichtsreich“ $\mathrm{zu}$ Aktivierende, in die Ressourcen und Zeit investiert werden und sind so auf sich selbst zurückgeworfen. Subjekte dieses Typus artikulieren häufig ihre Wahrnehmung, nicht (mehr) als erwerbskompetente Personen wahrgenommen zu werden - eine Einschätzung, die sich vielfach mit den in Hintergrundgesprächen und se- 
parat durchgeführten Interviews mit AMS-BeraterInnen erhobenen Einschätzungen und Fallbeschreibungen deckt. Außerdem fallen besonders in diesem Typenbereich sinnlose und passungenaue, insofern ,halbherzige“ Maßnahmen auf, in die Arbeitslose gedrängt werden - etwa Fortgeschrittenenenglischkurse für Handwerker ohne Basiskenntnisse u. Ä. Allenthalben konstituiert sich im Wechselspiel institutioneller Anrufung bzw. „Verarbeitung“ und den resignativen Selbstwahrnehmungen der Subjekte eine Gemengelage von Faktoren, die die gelungene Reintegration in den Arbeitsmarkt oder aber die Gestaltung eines sinnstiftenden Lebensentwurfes jenseits regulärer Erwerbsarbeit konterkarieren.

So zum Beispiel der 1952 geborene Herr Borske. Herr Borske ist in einer Großund Universitätsstadt im Nordwesten Deutschland geboren und verbrachte dort den Großteil seines (Arbeits-)Lebens. Er kommt aus einer politisch reflektierten Familie mit bildungsbürgerlichem Hintergrund (Vater: Opernsänger, Mutter: Hausfrau) mit einer acht Jahre früher geborenen Schwester. Nach abgeschlossener Hauptschule absolviert er zwei Berufsausbildungen, zum Tischler und Möbelkaufmann, und arbeitet im Anschluss einige Jahre als Angestellter im Möbelkaufhaus. Während dieser Zeit verliert er bei einem Unfall sein rechtes Auge, was seine Erwerbstätigkeit zunächst nicht beeinträchtigt. 1977 macht er sich dann im Innenausbau mit einer eigenen Firma selbstständig, die er betreibt, bis er 1998 seine schlecht laufende Firma aufgibt, seine aus Polen stammenden Frau heiratet, mit ihr nach Polen zieht und einen Sohn bekommt. Dort verdingt er sich mit dem Schmuggel von Elektrogeräten für das von seiner Frau eröffnete Geschäft. Auch dieses Projekt ist mäßig erfolgreich, sodass die Familie 2005 mit Hoffnung auf bessere Zukunftsperspektiven in eine Tourismusregion in Österreich zieht. Dort versucht er als Tischlerangestellter beruflichen Anschluss zu finden, was jedoch mittelfristig misslingt: Als mittlerweile 53Jähriger, zum Teil durch Rückenprobleme und sein fehlendes Auge Beeinträchtigter, kann er im Tischlereibetrieb, in dem er zunächst Arbeit findet, nur Hilfsarbeiten verrichten, was sein Selbstverständnis als gut ausgebildeter und erfahrener Handwerker mit einer gewissen Berufswürde untergräbt. Zudem macht ihm die mangelnde Akzeptanz als Deutscher unter Einheimischen zu schaffen, sodass er schließlich die Arbeit in seinem erlernten Beruf einvernehmlich aufgibt. Seither ist Herr Borske arbeitslos, mittlerweile auf die Mindestsicherung angewiesen, und versorgt die Familie zuhause, während die Frau ihrem seit dem Umzug nach Österreich ausgeübten Job als Hotelangestellte nachgeht. Das Aufgeriebensein zwischen der Versorgungsaufgabe, insbesondere für den noch schulpflichtigen Sohn, und der mangelnden Anschlussfähigkeit am Arbeitsmarkt bedingt latente Sinnkrisen und den Verlust von Zukunftshorizonten, wie sie schon bei den Arbeitslosen von Marienthal beobachtet wurden:

Das IST es ja (UNV.). (HEBT STIMME) Ich sitz ja nicht einfach sa//da, sa//und sage, d//du taugst ja sowieso nicht mehr (UNV.), dich will sowieso keiner. Aber dann, dann, dann frag' ich mich, was könnte ich noch machen? Ne, (HEBT STIMME) ich HAbe mich beWORben auch mal, äääh, als, als, als FAHrer, als, (UNV.). Das kann ja egal welche Auslieferungsfahrten sein, oder, oder äh irgendein Kleintransport oder irgendwas - jedes Mal wird es mir dann gesagt äh oder wird mir gesagt, ja äh wir suchen JÜNgere Leute, wir suchen 
jüngere Leute, ne. Das ist das Problem. (HEBT STIMME) Ich sehe HIER, für MICH keine richtige oder große Perspektive (SCHWERMÜTIG). * Seh' ich nich'. LEIder. * Wenn - wenn ich zwanzig, fünfundzwanzig Jahre jünger wäre, wäre das trotz, trotz dem AUge glaub' ich äh wäre das kein Problem. $\mathrm{Ne}$.

Insbesondere Alter und Krankheit verengen also die Perspektive auf die Möglichkeit sinnstiftender Arbeit und dadurch das Gelingen einer selbstbestimmten Lebensführung. Seine erklärte Aktivitätsbereitschaft (Ich sitz ja nicht einfach sal/da, sal/und sage, d//du taugst ja sowieso nicht mehr (UNV.), dich will sowieso keiner.) erfährt am Arbeitsmarkt keine Anerkennung. Die Zurückweisung hinterlässt Herrn Borske rat- und zukunftslos (Aber dann, dann, dann frag' ich mich, was könnte ich noch machen?). Die selbstverständlich beanspruchten Meriten eines verdienten und mehr oder weniger erfolgreichen und würdevollen Erwerbslebens bleiben ihm selbst mit einer Anspruchsnivellierung und flexibler Preisgabe des erlernten Berufes (ich HAbe mich beWORben auch mal, äääh, als, als, als FAHrer, . . . Das kann ja egal welche Auslieferungsfahrten sein, oder, oder äh irgendein Kleintransport oder irgendwas) - verwehrt. Statt wohlverdientem Ruhestand als gelungenes Ende eines gelungenen Erwerbslebens bleibt ihm nur der prekäre und noch dazu als gesellschaftlich illegitim geltende alimentierte „Ruhestand“.

In Folge haben betroffene Subjekte wie Herr Borske oft keine intrinsische Motivation (mehr), ihre geringe Energie und Hoffnung in wenig erfolgversprechende Versuche zu investieren, den Übertritt (zurück) in den regulären Arbeitsmarkt zu bewältigen und/oder sich in vorbereitenden Maßnahmen zu engagieren. Das Verhältnis zwischen Subjekt und Institutionen ist geprägt von wechselseitigem resignierten Laissez-faire. Die Menschen werden nicht mehr als zur arbeitsmarktlichen Sorge qualifizierte Subjekte adressiert und insofern desubjektiviert, dabei schließlich deaktiviert, da ihnen eine subjekthafte Teilhabe in erwerbsarbeitszentrierten Gesellschaften verwehrt wird. Insofern handelt sich meist nicht nur um Erwerbskrisen, sondern auch um Subjektivitäts- und Biografiekrisen in einem umfassenden Sinn.

Damit soll nicht ausgeschlossen werden, dass Desubjektivierungsprozesse auch ermöglichend sein können (vgl. Traue und Pfahl 2012). In unseren Fällen dominieren jedoch die verletzenden, einschränkenden und ausschließenden Dynamiken der Desubjektivierung. Diese betreffen einerseits die Ebene des Körpers in Beschreibungen von Erschöpfung und Depression (vgl. Ehrenberg 2004) bei den Befragten, andererseits auch die Ebene der sozialen Rechte. Das Gefühl von politischer Machtund Einflusslosigkeit bewirkt in unseren Fällen sowohl Rückzug und Ängstlichkeit (vgl. Bude 2014) als auch vermeintliche Identitätsstabilisierung basierend auf xenophober Abwertung oder anderer Mechanismen des „Otherings“, beispielsweise der Differenzierung zwischen ,würdigen“ und ,unwürdigen“ Armen. In dieser Sphäre bewegen sich - oder verharren - vor allem ältere, körperlich und/oder psychisch beeinträchtigte Menschen von meist niedrigem formalen Bildungsstatus oder auch (vor allem) Frauen, die durch Scheidung oder Trennung eines „,male breadwinners“ unvermittelt vor Existenznöten stehen, auf Transferleistungen angewiesen, schließlich aber nicht vermittelbar sind. 
Der vierte und letzte Idealtypus, „,die Alternativen “, der sich aus den empirischen Erkenntnissen konstruieren lässt, befindet sich am äußersten Pol des linken unteren Quadranten. Auch die ihm zuordenbaren Fälle haben die systemische Sphäre verlassen, allerdings nicht in Richtung phlegmatischer Resignation wie Typ 3, sondern in einen Bereich kreativer Selbstermächtigung. So berichtet uns Herr Wolf über sein ungemeldetes, unversteuertes, insofern illegales Online-Poker-Geschäft. Herr Wolf wurde 1986 als ältestes von drei Geschwistern im Westen Österreichs geboren, die geschiedenen Eltern, beide ungelernt, arbeiten in prekären Jobs. Er zieht mit 15 aus dem Elternhaus aus und lebt, jugendwohlfahrtlich betreut, alleine. Nach Abschluss der Pflichtschule ist Herr Wolf unschlüssig, was seine Zukunft betrifft; auf Jobsuche wird er vom AMS betreut und in eine Kochlehre vermittelt. Schnell merkt er, dass ihm dieser Berufsweg nicht entspricht (fühlt sich fehlberaten), und beendet die Ausbildung vorzeitig. Es folgt eine über zehn Jahre andauernde Episode von Gelegenheitsarbeiten, Abbrüchen und Instabilität. Herr Wolf kann auf dem regulären Arbeitsmarkt kein kontinuierliches Einkommen generieren; das wiegt umso schwerer, als Schulden aus einem jugendlichen Vergehen Herrn Wolfs Alltag und Zukunft überschatten. Nicht zuletzt würde ein reguläres Einkommen bis auf den Mindestsatz gepfändet werden. So beginnt er, seiner Leidenschaft für digitale Kommunikation und Arbeit, die er am Arbeitsmarkt nicht leben kann, privat nachzugehen - und lernt schnell, wie er damit vorbei an Finanzamt und AMS Geld verdienen kann. Vom hobbymäßigen Kartenspiel im Internet wird bald eine großangelegte OnlinePoker-Plattform, die er kommerziell betreibt:

.. . war dann eigentlich mit achtzehn hab' ich schon eine Bankroll, die man mit Freerolls zusammen * gespielt hab' halt gehabt, aaahm, die ist mir dann eingezogen worden, nachdem ich auszahlen wollte, das erste Mal, weil ich einen Ausweis hingeschickt hab', und ich ursprünglich falsche Angaben hab' (LAUTER), also noch mal Spiel von vorne, ääähm hab' dann zzzu//binnen kürzstester Zeit eigentlich genug Geld gehabt, dass ich wieder in den höheren Stakes gespielt hab', * hab' von achtzehn bis einundzwanzig vom Pokern gelebt, * mehr oder weniger gelebt, also so ein Tausender im Monat ist schon rausgesprungen, * ääähm ** da hab' ich dann aber irgendwann einmal gesehen, okay, WENN ich vom Pokern leben will, dann muss ich entweder jeden Tag vier, fünf Stunden spielen, und das brennt extrem aus, wenn man zwölf oder sechzehn oder zwanzig Tische gleichzeitig spielt. *3* Uuund ich hab' halt nebenbei immer schon einen Poker-Blog gehabt, uuund oder halt gleich einmal \#am Anfang .... \#

Herr Wolf beginnt also, aus einer Leidenschaft findig ein Geschäft zu etablieren. Zunächst noch etwas holprig und experimentell (und ich ursprünglich falsche Angaben hab'), aber frustresistent und lernfähig (also noch mal Spiel von vorne, ääähm hab' dann zzzu//binnen kürzester Zeit eigentlich genug Geld gehabt, dass ich wieder in den höheren Stakes gespielt hab'), investiert er Zeit und Energie, sich den erwählten Nischenbereich autodidaktisch anzueignen und gleichsam spielerisch $\mathrm{zu}$ durchdringen. Bald bemerkt er, dass er damit ein konkretes Einkommen lukrieren kann, und macht das Pokern zum klandestinen Vollzeit-,,Job“. Er mausert sich zum Experten in einem hochschwelligen Bereich jenseits normativer, regulier- 
ter Arbeitssphären und reüssiert durchaus auch ökonomisch. Insofern begreifen wir auch ihn und den von ihm repräsentierten Typus als ,,aktiviert“", dabei aber weniger zum Normalarbeitssubjekt, denn vielmehr zum kreativen „Do-It-Yourself“-Subjekt, zur „Lebenskünstlerin“. Typisch für Fälle sind etwa autodidaktische Aneignungen spezieller Kompetenzen oder sehr spezifischen ExpertInnentums, informelle (zum Teil auch niederschwellig kriminelle) Erwerbsformen etc. Transferleistungen dienen hier weniger zur Überbrückung, denn zur Ermöglichung einer mehr oder weniger autonomen Selbstentwicklung abseits des Sozialsystems. In diesem Quadranten beobachten wir insbesondere jüngere, tendenziell formal gut gebildete Personen, die ihren Bildungsweg entweder noch nicht abgeschlossen haben oder deren Lebensgeschichte Situationen und Brüche beinhaltet, die kreative Anpassungsbemühungen erfordern oder voraussetzen. Letzteres betrifft etwa MigrantInnen oder Subjekte mit Devianz-(etwa Haft-)erfahrungen.

\section{Resümee}

Auf Ebene der von uns rekonstruierten Handlungsmuster zeigen sich in den untersuchten Fällen, in starkem Kontrast zu alltagsweltlichen und medialen Stereotypen von LeistungsempfängerInnen oder „Armen“ in der berüchtigten (sozialen) „Hängematte", durchwegs Subjekte, die im Gegenteil getrieben sind von permanenter Aktivität. Tätigsein in einem umfassenden Sinn charakterisiert das Gros der Fälle. Viele der Menschen in Armutslagen sind unablässig damit beschäftigt, sich andauernd aktualisierende biografische Krisen zu bewältigen, ohne sie tatsächlich nachhaltig lösen zu können.

Das Alltagsleben ist in den meisten Fällen geprägt von Überforderung und dem permanenten Druck, es in seinen basalen Funktionen finanziell, zeitlich und organisatorisch aufrecht $\mathrm{zu}$ erhalten - dabei aber ohne sinnstiftende Anerkennung und Selbstverwirklichungs- und -bestimmungspotential. Darin knüpfen unsere Ergebnisse an jene von Dörres et al. (2006, 2008) und das dort beschriebene „(Des)Integrationsparadoxon nachfordistischer Arbeitsgesellschaften“ an, demzufolge ,die handelnden Subjekte immer wieder zu eigensinnigen Bearbeitungen ihrer Problemlagen“ gezwungen sind und prekäre Beschäftigung und Arbeitslosigkeit „so - zumindest kompensatorische - Re-Integrationsanstrengungen“ fördern (Dörre et al. 2008, S. 7). Der aktivierende Sozialstaat produziert also gewissermaßen aktive Subjekte, deren Aktivität er in den meisten Fällen aber zugleich selbstbezüglich auf das Bearbeiten und Lösen strukturell (sozialpolitisch) perpetuierter Krisen limitiert und so biografische Krisen befördert. Denselben Umstand beschreibt die Wiener Studie zur Bedarfsorientierten Mindestsicherung als „Aktivierung ohne Arbeit“" (L\&R Sozialforschung 2011, S. 148).

Die untersuchten Subjekte sind also in den meisten Fällen „aktiv“, das bedeutet aber nicht, dass sie in Richtung des sozialpolitischen Ziels der „Employability“ aktiviert wurden. Trotz ihrer gemeinsamen Lage - als Angewiesene auf Transferleistungen - handeln sie vor dem Hintergrund ihrer unterschiedlichen Lebenssituationen, Biografien und Lebenspläne. Wie in den Armutsdynamiken-Studien der 90er-Jahre bereits gezeigt wurde (vgl. Leibfried et al. 1995), gibt es nicht „den Arbeitslosen“ 
und ,die Arme“, sondern Armut zeigt sich vielgestaltig - transitorisch oder dauerhaft - und multidimensional.

Dass eine „one-size-fits-all“-Maßnahme vor dem Hintergrund der Vielgestaltigkeit (post-/zweit-)moderner Lebensformen nicht bei allen die gleiche Handlungsaktivierung auslöst und unintendierte Nebeneffekte zur Folge hat, zeigen unsere Untersuchungen augenscheinlich. Dass nicht jedeR unabhängig von den äußeren Bedingungen gleichermaßen aktiviert werden kann, ebenso. Die diskutierten Fälle zeigen vielmehr, dass eine selbstbestimmte, nachhaltige Überwindung von Armutslagen in den meisten Fällen verhindert und stattdessen der Rückzug in Resignation, der Ausstieg aus dem formellen Erwerbstätigkeitsleben oder das passive Prozessiertwerden zwischen Sozialverwaltung und prekären Arbeitsverhältnissen befördert wird. In den wenigsten Fällen werden die betroffenen Subjekte gemäß den aktivierungspolitischen Zielen zur „Employability“ aktiviert und in stabile, längerfristige, nicht-prekäre Arbeitsverhältnisse vermittelt. Bei den unbedingt „Arbeitswilligen“ scheitert die Vermittlung und Aktivierung entweder am arbeitsmarktlichen Angebot und der Anerkennung von Ausbildungsabschlüssen - das ist häufig der Fall bei den relativ hoch gebildeten, oft migrantischen Arbeitslosen - oder am Fehlen von inklusiven Arbeitsverhältnissen, wie es an kranken oder sog. ,lernbehinderten“ oder „entwicklungsverzögerten“ TransferleistungsempfängerInnen deutlich wird, die im Sinne des aktivierungspolitischen „Arbeitsfähigkeitsbegriffes“ zwar drei Stunden arbeiten können, dafür aber keine passenden Arbeitsverhältnisse vorfinden. Gezeigt werden konnte an den dargestellten Handlungsaktivierungstypen auch, dass gegenüber individualisierender, aktivierender Sozialpolitik individualisierende Handlungsstrategien entwickelt werden und nicht etwa kollektive wie die Organisation zu Solidaritätsgemeinschaften, Netzwerken usw.

Für das Verhältnis zwischen aktivierungspolitischer sozialpolitischer Steuerung und Aneignung bedeutet das, dass Institutionen und Diskurse zwar als Zumutungsund Bewältigungskontexte, in den seltensten Fällen aber auch als Ermöglichungskontexte betrachtet werden können - und damit einerseits für die Überwindung von Armutslagen, andererseits für die Herstellung von Employability als dysfunktional zu charakterisieren sind.

Die Frage, ob es sich dabei um allgemeine Bewältigungsstrategien von Arbeitslosen handelt, kann nur anhand von historischen sowie gegenwärtigen Vergleichsstudien beantwortet werden, solchen, die Arbeitslose unter anderen sozialpolitischen Regimes untersuchen. Im Vergleich zu den Studien der Arbeitslosen von Marienthal zeigt sich in unserer Studie eine höhere Aktivität der Langzeitarbeitslosen, allerdings nicht im Sinne des sozialpolitischen Ziels der ,employability“. Im Vergleich zu den Studien, die in Deutschland unter dem Aktivierungsregime durchgeführt wurden, insbesondere der von Weißmann (2016), überwiegen in unserem Sample trotz der eigensinnigen Aneignungen und dem hohen Aktivitätslevel die wahrgenommenen Autonomieverluste und Entmächtigungsgefühle der langzeitarbeitslosen MindestsicherungsempfängerInnen.

Funding Open access funding provided by University of Innsbruck and Medical University of Innsbruck.

Open Access Dieser Artikel wird unter der Creative Commons Namensnennung 4.0 International Lizenz (http://creativecommons.org/licenses/by/4.0/deed.de) veröffentlicht, welche die Nutzung, Vervielfäl- 
tigung, Bearbeitung, Verbreitung und Wiedergabe in jeglichem Medium und Format erlaubt, sofern Sie den/die ursprünglichen Autor(en) und die Quelle ordnungsgemäß nennen, einen Link zur Creative Commons Lizenz beifügen und angeben, ob Änderungen vorgenommen wurden.

\section{Literatur}

Atzmüller, Roland. 2009. Die Entwicklung der Arbeitsmarktpolitik in Österreich. Dimensionen von Workfare in der österreichischen Sozialpolitik. Kurswechsel 2009(4):24-34.

Atzmüller, Roland, Manfred Krenn, und Ulrike Papouschek. 2012. Innere Aushöhlung und Fragmentierung des österreichischen Modells: Zur Entwicklung von Erwerbslosigkeit, prekärer Beschäftigung und Arbeitsmarktpolitik. In Neue Prekarität. Die Folgen aktivierender Arbeitsmarktpolitik - europäische Länder im Vergleich, Hrsg. Karin Scherschel, Peter Streckeisen, und Manfred Krenn, 75-109. Frankfurt am Main, New York: Campus.

Bosančić, Saša. 2014. Arbeiter ohne Eigenschaften. Über die Subjektivierungsweisen angelernter Arbeiter. Wiesbaden: Springer VS.

Bosančić, Saša. 2016. Zur Untersuchung von Subjektivierungsweisen aus wissenssoziologisch-diskursanalytischer Perspektive. Methodologische Überlegungen. In Perspektiven wissenssoziologischer Diskursforschung, Hrsg. Saša Bosančić, Reiner Keller, 95-119. Wiesbaden: Springer VS.

Bothfeld, Silke, und Sigrid Betzelt. 2014. Autonomie - ein neues Leitbild einer modernen Arbeitsmarktpolitik. WISO direkt. http://library.fes.de/pdf-fi-les/wiso/10955.pdf (Erstellt: 10.2014). Zugegriffen: 14. Okt. 2016. Friedrich-Ebert-Stiftung.

Bröckling, Ulrich. 2007. Das unternehmerische Selbst. Soziologie einer Subjektivierungsform. Frankfurt am Main: Suhrkamp.

Bude, Heinz. 2014. Gesellschaft der Angst. Hamburg: Hamburger Edition.

derStandard.at. 2016. Stöger kritisiert ,ppopulistische Aussagen“ zur Mindestsicherung. http://derstandard. at/2000039466097/Stoeger-kritisiert-populistische-Aussagen-zur-Mindestsicherung (Erstellt: 21. Juni 2016). Zugegriffen: 1. Aug. 2016.

Dörre, Klaus, Klaus Kraemer, und Frederic Speidel. 2006. Prekäre Beschäftigung und soziale Desintegration - Ursprünge, Konsequenzen und politische Verarbeitungsformen unsicherer Erwerbsarbeit. Jahrbuch Arbeit, Bildung, Kultur, Bd. 23/24, 9-40. Recklinghausen: FIAB Verlag.

Dörre, Klaus, Peter Bescherer, Silk Röbenack, und Karen Schierhorn. 2008. Eigensinnige ,Kunden ': Auswirkungen strenger Zumutbarkeitsregeln auf Langzeitarbeitslose und prekär Beschäftigte. SFB 580 Mitteilungen 26:10-42.

du Gay, Paul, und Alan Scott. 2010. State transformation or regime shift? Addressing some confusions in the theory and sociology of the state. Sociologica 2:1-23. https://doi.org/10.2383/32707.

Dux, Günter. 2008. Warum denn Gerechtigkeit. Die Logik des Kapitals. Die Politik im Widerstreit mit der Ökonomie. Weilerswist: Velbrück Wissenschaft.

Dux, Günter. 2009. Von allem Anfang an: Macht, nicht Gerechtigkeit. Studien zur Genese und historischen Entwicklung des Postulats der Gerechtigkeit. Weilerswist: Velbrück Wissenschaft.

Ehrenberg, Alain. 2004. Das erschöpfte Selbst. Depression und Gesellschaft in der Gegenwart. Frankfurt am Main: Campus.

Europäischer Rat. 2000. Schlussfolgerungen des Vorsitzes. Europäischer Rat (Lissabon) 23. und 24. März 2000. Lissabon: Europäischer Rat.

Foucault, Michel. 1984. Sexualität und Wahrheit 3: Die Sorge um sich. Frankfurt am Main: Suhrkamp.

Foucault, Michel. 1993. About the Beginning of the Hermeneutics of the Self. Political Theory 21:198-227

Foucault, Michel. 2010. Kritik des Regierens. Schriften zur Politik. Berlin: Suhrkamp.

Le Galès, Patrick, und Alan Scott. 2009. Die Wiederherstellung des Marktsubjekts. Berliner Journal für Soziologie 19:6-28.

Geimer, Alexander. 2014. Das authentische Selbst in der Popmusik - Zur Rekonstruktion von diskursiven Subjektfiguren sowie ihrer Aneignung und Aushandlung mittels der Dokumentarischen Methode. Österreichische Zeitschrift für Soziologie 39(2):111-130.

Glaser, Barney G., und Anselm L. Strauss. 1967. The discovery of grounded theory. Strategies for qualitative research. New Brunswick, London: AldineTransaction.

Globisch, Claudia. 2012. Strukturwandel sozialpolitischer Steuerung. In Markt - Inklusion - Gerechtigkeit. Zum Problem der sozialen Gerechtigkeit in der Marktgesellschaft. Sonderheft 11/2012 der Österreichischen Zeitschrift für Soziologie, Hrsg. Gerda Bohmann, Heinz-Jürgen Niedenzu, 133-154. Wiesbaden: Springer VS. 
Globisch, Claudia. 2013. Radikaler Antisemitismus. Inklusions- und Exklusionssemantiken von links und rechts in Deutschland. Wiesbaden: Springer VS.

Globisch, Claudia. 2016. Zwischen Autonomie und Heteronomie. Die Arbeitslosen der Aktivierungsgesellschaft. Habilitationsschrift, unveröff. Manuskript

Grimm, Natalie, Andreas Hirseland, und Berthold Vogel. 2013. Die Ausweitung der Zwischenzone. Erwerbsarbeit im Zeichen der neuen Arbeitsmarktpolitik. Soziale Welt 64(3):249-268.

Habermas, Jürgen. 1998. Die postnationale Konstellation: Politische Essays. Frankfurt am Main: Suhrkamp.

Hibou, Béatrice. 2015. The Bureaucratization of the World in the Neoliberal Era. An International and Comparative Perspective. Basingstoke: Palgrave Macmillan.

Hirseland, Andreas, und Philipp Ramos Lobato. 2010. Armutsdynamik und Arbeitsmarkt. Entstehung, Verfestigung und Überwindung von Hilfebedürftigkeit bei Erwerbsfähigen. IAB-Forschungsbericht 03/2010. Nürnberg: IAB.

Hirseland, Andreas, und Philipp Ramos Lobato. 2012. Zwischen „Hartz IV“ und geförderter Beschäftigung. Positionierung und Teilhabeerleben im reformierten Sozialstaat. In Wechselverhältnisse im Wohlfahrtsstaat. Dynamiken gesellschaftlicher Justierungsprozesse, Hrsg. Mechthild Bereswill, Carmen Figlestahler, Marko Perels, Lisa Yashodhara Haller, und Franz Zahradnik, 273-291. Münster: Westfälisches Dampfboot.

Hirseland, Andreas, Markus Promberger, und Ulrich Wenzel. 2007. Armutsdynamik und Arbeitsmarkt. Qualitative Beobachtungen und Befragungen im Feld von Arbeitsmarkt und sozialer Sicherung. In Neue Daten für die Sozialstaatsforschung. Zur Konzeption der IAB-Panelerhebung „Arbeitsmarkt und Soziale Sicherung “ IAB Forschungsbericht 12/2007., Hrsg. Markus Promberger, 102-130. Nürnberg: IAB.

Holz, Klaus. 2001. Nationaler Antisemitismus. Wissenssoziologie einer Weltanschauung. Hamburg: Hamburger Edition.

Jahoda, Marie, Paul F. Lazarsfeld, und Hans Zeisel. 1975. Die Arbeitslosen von Marienthal. Ein soziographischer Versuch über die Wirkungen langandauernder Arbeitslosigkeit. Frankfurt am Main: Suhrkamp.

Keller, Reiner. 2011. Diskursforschung. Eine Einführung für SozialwissenschaftlerInnen. Wiesbaden: VS.

Keller, Reiner, Werner Schneider, und Willy Viehöfer. 2012. Theorie und Empirie der Subjektivierung in der Diskursforschung. In Diskurs - Macht - Subjekt. Theorie und Empirie von Subjektivierung in der Diskursforschung, Hrsg. Reiner Keller, Werner Schneider, und Willy Viehöfer, 7-20. Wiesbaden: VS.

Klammer, Ute, und Simone Laiber. 2004. Aktivierung und Eigenverantwortung in europäisch-vergleichender Perspektive. Berlin: Hans-Böckler-Stiftung.

L\&R Sozialforschung. 2011. Erwerbspotenzial von SozialhilfebezieherInnen in Wien. Bestandsaufnahme vor Einführung der bedarfsorientierten Mindestsicherung. Wien: L\&R Spezialforschung.

Leibfried, Stephan, Lutz Leisering, Petra Buhr, Monika Ludwig, Eva Mädje, Thomas Olk, Wolfgang Voges, und Michael Zwick. 1995. Zeit der Armut. Lebensläufe im Sozialstaat. Frankfurt am Main: Suhrkamp.

Lemke, Thomas, Ulrich Bröckling, und Susanne Krasmann (Hrsg.). 2000. Gouvernementalität der Gegenwart. Studien zur Ökonomisierung des Sozialen. Frankfurt am Main: Suhrkamp.

Lessenich, Stephan. 2008. Die Neuerfindung des Sozialen. Der Sozialstaat im flexiblen Kapitalismus. Bielefeld: transcript.

Lessenich, Stephan. 2012. Das Anerkennungsdefizitsyndrom des Wohlfahrtsstaats. In Markt - Inklusion - Gerechtigkeit. Zum Problem der sozialen Gerechtigkeit in der Marktgesellschaft. Sonderheft 11/2012 der Österreichischen Zeitschrift für Soziologie, Hrsg. Gerda Bohmann, HeinzJürgen Niedenzu, 133-154. Wiesbaden: Springer VS.

Ludwig-Mayerhofer, Wolfgang, Olaf Behrend, und Ariadne Sondermann. 2009. Auf der Suche nach der verlorenen Arbeit. Arbeitslose und Arbeitsvermittler im neuen Arbeitsmarktregime. Konstanz, München: UVK.

Oevermann, Ulrich. 2000. Die Methode der Fallrekonstruktion in der Grundlagenforschung sowie der klinischen und pädagogischen Praxis. In Die Fallrekonstruktion. Sinnverstehen in der sozialwissenschaftlichen Forschung, Hrsg. Klaus Kraimer, 58-156. Frankfurt am Main: Suhrkamp.

Oevermann, Ulrich, Tilman Allert, Elisabeth Konau, und Jürgen Krambeck. 1979. Die Methodologie einer ,objektiven Hermeneutik“ und ihre allgemeine forschungslogische Bedeutung in den Sozialwissenschaften. In Interpretative Verfahren in den Sozial- und Textwissenschaften, Hrsg. Hans-Georg Soeffner, 352-434. Stuttgart: Metzler. 
Pfahl, Lisa, Schürmann, Lena, Traue, Boris. 2015. Das Fleisch der Diskurse. Zur Verbindung von Biographie- und Diskursforschung in der wissenssoziologischen Subjektivierungsanalyse am Beispiel der Behindertenpädagogik. In Diskursanalytische Zugänge zu Bildungs- und Erziehungsverhältnissen, Hrsg. Susann Fegter, Fabian Kessel, Antje Langner, Marion Ott, Daniela Rothe und Daniel Wrana, 89-106. Wiesbaden: VS.

Przyborski, Aglaja, und Monika Wohlrab-Sahr. 2010. Qualitative Sozialforschung. Ein Arbeitsbuch, 2. Aufl., München: Oldenbourg.

Rat der Europäischen Union. 2001. Europäische Sozialagenda, auf der Tagung des Europäischen Rates in Nizza am 7., 8. und 9. Dezember 2000 angenommen. Luxembourg: Rat der Europäischen Union.

Rose, Nikolas. 1996. The death of the social: re-figuring the territory of government. British Journal of Sociology 43:173-205.

Schreiber-Barsch, Silke, und Lisa Pfahl. 2014. Zur Funktion der ,Anderen“: Klassifizierungspraktiken im Bildungswesen und das System des Lebenslangen Lernens. Hessische Blätter für Volksbildung 3:232-237.

Schütze, Fritz. 1984. Kognitive Figuren des autobiographischen Stegreiferzählens. In iographie und soziale Wirklichkeit: Neue Beiträge und Forschungsperspektiven, Hrsg. Martin Kohli, Robert Günther, 78-117. Stuttgart: Metzler.

Scott, Alan, und Richard Weiskopf. 2008. Freedom and constraint under a „Neoliberal“ regime of choice. In Macro approaches Sage Handbook of Organizational Behaviour, Bd. 2, Hrsg. Cary L. Cooper, Stewart R. Clegg, 68-84. London: SAGE.

Streeck, Wolfgang. 2013. Gekaufte Zeit. Die vertagte Krise des demokratischen Kapitalismus. Berlin: Suhrkamp.

Traue, Boris, und Lisa Pfahl. 2012. Desubjektivierungen. Zum Verhältnis von Körper, Wissen und Recht nach dem Neoliberalismus. In Wechselverhältnisse im Wohlfahrtsstaat. Dynamiken gesellschaftlicher Justierungsprozesse, Hrsg. Mechthild Bereswill, Carmen Figlestahler, Lisa Yashodhara Haller, Marko Perels, und Franz Zahradnik, 124-136. Münster: Westfälisches Dampfboot.

Vobruba, Georg. 1989. Arbeiten und Essen. Politik an den Grenzen des Arbeitsmarkts. Wien: Passagen.

Vobruba, Georg. 2006. Entkopplung von Arbeit und Einkommen. Das Grundeinkommen in der Arbeitsgesellschaft. Wiesbaden: VS.

Weisenbacher, Uwe. 1993. Moderne Subjekte zwischen Mythos und Aufklärung. Pfaffenweiler: Centaurus Verlag.

Weißmann, Marliese. 2016. Dazugehören. Handlungsstrategien von Arbeitslosen. Konstanz, München: UVK.

Weißmann, Marliese, und Kornelia Sammet. 2010. Individueller Anspruch versus erzwungene Gemeinschaft: Auswirkungen des Verwaltungshandelns auf Biographie und Lebensführung von ALG-IIEmpfängern am Beispiel der „Bedarfsgemeinschaft“. BIOS 23(1):28-46.

Wenzel, Ulrich. 2008. Fördern und Fordern aus Sicht der Betroffenen: Verstehen und Aneignung sozialund arbeitsmarktpolitischer Maßnahmen des SGB II. Zeitschrift für Sozialreform (ZSR) 54(1):57-78.

Zirra, Sascha. 2010. Die Europäisierung nationaler Beschäftigungsstrategie. Europäische Koordinierung und institutionelle Reformen. Wiesbaden: VS.

Claudia Globisch ist Assistenzprofessorin am Institut für Soziologie der Universität Innsbruck. Sie habilitiert zum Thema „Autonomie und Heteronomie: Die Arbeitslosen der Aktivierungsgesellschaft“. Ihre Forschungsschwerpunkte sind Armuts- und Ungleichheitsforschung, Soziologie der Sozialpolitik, Rechtsextremismus- und Antisemitismusforschung, Wissenssoziologie und Qualitative Methoden (spez. Subjektivierungsforschung, Semantik- und Diskursanalysen \& Biographieforschung). Ausgewählte Publikationen: „Radikaler Antisemitismus. Inklusions- und Exklusionssemantiken von links und rechts in Deutschland“, Wiesbaden: VS Verlag (2013), Relationale Autonomie und Sozialpolitik - eine Soziologie der Kritik, in: Börner et. al. (Hrsg.): Praktiken der Selbstbestimmung. Zwischen subjektivem Anspruch und institutionellem Funktionserfordernis. Mit einem Vorwort von Hartmut Rosa, Wiesbaden: VS Verlag (2017).

Fabian Madlung studierte Politikwissenschaft und Soziologie in Innsbruck. Am Innsbrucker Institut für Soziologie arbeitet er an Projekten zur Erforschung prekärer Lebenslagen, Armut und sozialpolitischer Interventionen. Seine Schwerpunkte liegen außerdem im Bereich qualitativer Methodologie. 